\title{
AN EQUICONVERGENCE THEOREM FOR A PAIR OF SECOND ORDER DIFFERENTIAL EQUATIONS
}

\author{
BIKAN BHAGAT
}

ABSTRACT. The asymptotic solutions of a matrix differential equation of the second order have been considered and then an equiconvergence theorem has been proved.

1. Let $L$ denote the matrix operator

$$
L \equiv\left(\begin{array}{cc}
\frac{d^{2}}{d x^{2}}+p(x) & r(x) \\
r(x) & \frac{d^{2}}{d x^{2}}+q(x)
\end{array}\right),
$$

and $\phi$ a vector having two components $u \equiv u(x)$ and $v \equiv v(x)$ represented as a column matrix $\left(\begin{array}{l}u \\ v\end{array}\right)$.

Then consider the matrix equation

$$
(L+\lambda) \phi=0 \text {. }
$$

The main object of the paper is to obtain an equiconvergence theorem associated with the system (1.2), using the method of Titchmarsh [4, Chapter I] and Roos and Sangren [3]. Notations used by the author [1] will be used here.

The boundary conditions to be satisfied by $\phi(x)=\left(\begin{array}{l}u(x) \\ v(x)\end{array}\right)$ are given by

$$
\begin{aligned}
& M(a, \phi) \equiv a_{j 1} u(a)+a_{j 2} u^{\prime}(a)+a_{j 3} v(a)+a_{j 4} v^{\prime}(a)=0 \\
& N(b, \phi) \equiv b_{j 1} u(b)+b_{j 2} u^{\prime}(b)+b_{j 3} v(b)+b_{j 4} v^{\prime}(b)=0
\end{aligned}
$$

where (i) $a_{j k}$ and $b_{j k}$ are real valued constants;

Received by the editors March 23, 1970.

AMS 1969 subject classifications. Primary 3430, 3450, 3410.

Key words and phrases. Matrix operator, equiconvergence theorem, boundary value problem, selfadjoint, bilinear concomitant, boundary condition vectors, Wronskian, eigenvalue, integral equations, Conte and Sangren lemma, Fourier series, bounded variation, residue theorem.

(c) American Mathematical Society 1972 
(ii) the set $\left\{a_{1 k}\right\}$ is linearly independent of the set $\left\{a_{2 k}\right\}$, and the set $\left\{b_{1 k}\right\}$ is linearly independent of the set $\left\{b_{2 k}\right\}$;

(iii) at least two of the ratios $a_{1 k} / a_{2 k}(k=1,2,3,4)$ and two of the corresponding ratios $b_{1 k} / b_{2 k}(k=1,2,3,4)$ are unequal, say,

$$
\text { (iv) } \begin{aligned}
& a_{12} / a_{22} \neq a_{14} / a_{24}, \quad b_{12} / b_{22} \neq b_{14} / b_{24} ; \\
& b_{14} b_{23}-b_{24} b_{13}+b_{12} a_{21}-a_{11} a_{22}=0 \\
& b_{11} b_{22}=0 .
\end{aligned}
$$

The relations (1.4) ensure that the boundary value problem considered is selfadjoint. $p(x), q(x)$ and $r(x)$ are real valued and continuous in $a \leqq x \leqq b$.

The bilinear concomitant $[\phi \theta]$ of any two vectors

is defined by

$$
\phi=\left(\begin{array}{l}
u_{1} \\
v_{1}
\end{array}\right) \quad \text { and } \quad \theta=\left(\begin{array}{l}
u_{2} \\
v_{2}
\end{array}\right)
$$

$$
[\phi \theta]=u_{1}^{\prime} u_{2}-u_{1} u_{2}^{\prime}+v_{1}^{\prime} v_{2}-v_{1} v_{2}^{\prime} .
$$

If $\phi$ and $\theta$ are solutions of (1.2) for the same value of $\lambda$, then [ $\phi \theta]$ is independent of $x$ and is a function of $\lambda$ alone.

Let

$$
\phi_{j}(a \mid x, \lambda)=\left(\begin{array}{l}
u_{j}(a \mid x, \lambda) \\
v_{j}(a \mid x, \lambda)
\end{array}\right) \quad(j=1,2)
$$

and

$$
\phi_{k}(b \mid x, \lambda)=\left(\begin{array}{l}
u_{k}(b \mid x, \lambda) \\
v_{k}(b \mid x, \lambda)
\end{array}\right) \quad(k=3,4)
$$

be the boundary condition vectors at $x=a$ and $x=b$ respectively so that the boundary conditions (1.3) are written in the form

where

$$
\left[\phi(x, \lambda) \phi_{j}(a \mid x, \lambda)\right]=0 \quad \text { and } \quad\left[\phi(x, \lambda) \phi_{k}(b \mid x, \lambda)\right]=0
$$

$$
\left[\phi_{1} \phi_{2}\right]=0, \quad\left[\phi_{3} \phi_{4}\right]=0 .
$$

Conditions (1.6) are equivalent to conditions (1.4).

The Wronskian $W(\lambda)$ of the boundary condition vectors is given by

$$
W(\lambda)=\left[\phi_{1} \phi_{3}\right]\left[\phi_{2} \phi_{4}\right]-\left[\phi_{1} \phi_{4}\right]\left[\phi_{2} \phi_{3}\right]
$$

It is shown by the author [1] that the eigenvalues are the zeros of $W(\lambda)$; they are real and simple or double.

2. We now proceed to obtain results analogous to $\S 7$, Chapter I of Titchmarsh [4]. 
Lemma 2.1. Let

$$
\phi(\alpha \mid x, \lambda)=\left(\begin{array}{l}
u(\alpha \mid x, \lambda) \\
v(\alpha \mid x, \lambda)
\end{array}\right)
$$

be a solution of (1.2) such that $u(\alpha \mid \alpha, \lambda)=A, u^{\prime}(\alpha \mid \alpha, \lambda)=B, v(\alpha \mid \alpha, \lambda)=C$ and $v^{\prime}(\alpha \mid \alpha, \lambda)=D$. Then $\phi(\alpha \mid x, \lambda)$ satisfies the system of integral equations

$$
\begin{aligned}
& u(\alpha \mid x, \lambda)= A \cos \{\mu(x-\alpha)\}+(B / \mu) \sin \{\mu(x-\alpha)\} \\
&-\frac{1}{\mu} \int_{\alpha}^{x}\{p(y) u(\alpha \mid y, \lambda)+r(y) v(\alpha \mid y, \lambda)\} \times \sin \{\mu(x-y)\} d y, \\
& v(\alpha \mid x, \lambda)= C \cos \{\mu(x-\alpha)\}+(D / \mu) \sin \{\mu(x-\alpha)\} \\
&-\frac{1}{\mu} \int_{\alpha}^{x}\{r(y) u(\alpha \mid y, \lambda)+q(y) v(\alpha \mid y, \lambda)\} \\
& \times \sin \{\mu(x-y)\} d y,
\end{aligned}
$$

where $\lambda=\mu^{2}$.

The result follows on using (1.2) and then integrating by parts.

LEMMA 2.2. Let

and

$$
\phi_{j}(a \mid x, \lambda)=\left(\begin{array}{l}
u_{j}(a \mid x, \lambda) \\
v_{j}(a \mid x, \lambda)
\end{array}\right) \quad(j=1,2)
$$

$$
\phi_{k}(b \mid x, \lambda)=\left(\begin{array}{l}
u_{k}(b \mid x, \lambda) \\
v_{k}(b \mid x, \lambda)
\end{array}\right) \quad(k=3,4)
$$

be the boundary condition vectors for the system (1.2) and $\lambda=\mu^{2}$ where $\mu=\sigma+i$. Then for $|\mu| \geqq\left|\mu_{0}\right|$,

$$
\begin{aligned}
& \phi_{j}(a \mid x, \lambda)=O\left(e^{|t|(x-a)}\right) \quad(j=1,2), \\
& u_{j}(a \mid x, \lambda)=a_{j 2} \cos \{\mu(x-a)\}+O\left(e^{|t|(x-a)} /|\mu|\right) \text {, } \\
& v_{j}(a \mid x, \lambda)=a_{j 4} \cos \{\mu(x-a)\}+O\left(e^{|t|(x-a)} /|\mu|\right), \quad(j=1,2) \\
& \text { (2.5) (iii) } \phi_{k}(b \mid x, \lambda)=O\left(e^{|t|(b-x)}\right) \quad(k=3,4) \text {, } \\
& u_{k}(b \mid x, \lambda)=b_{j 2} \cos \{\mu(b-x)\}+O\left(e^{|t|(b-x)} /|\mu|\right) \text {, } \\
& v_{k}(b \mid x, \lambda)=b_{j 4} \cos \{\mu(b-x)\}+O\left(e^{|t|(b-x)} /|\mu|\right),
\end{aligned}
$$

when $k=3, j=1$ and when $k=4, j=2$.

Each of the results holds uniformly for $a \leqq x \leqq b$.

\section{Putting}

and

$$
u(\alpha \mid x, \lambda)=u_{j}(a \mid x, \lambda)=g_{j}(x) e^{|l|(x-a)}
$$

$$
v(\alpha \mid x, \lambda)=v_{j}(a \mid x, \lambda)=h_{j}(x) e^{|t|(x-a)}
$$


in (2.1) and (2.2) respectively, we get

$$
\begin{aligned}
g_{j}(x)= & a_{j 2} \cos \{\mu(x-a)\} e^{-|t|(x-a)}-\left(a_{j 1} / \mu\right) \sin \{\mu(x-a)\} e^{-|t|(x-a)} \\
& -\frac{1}{\mu} \int_{a}^{x}\left\{p(y) g_{j}(y)+r(y) h_{j}(y)\right\} e^{-|t|(x-y)} \sin \{\mu(x-y)\} d y, \\
h_{j}(x)= & a_{j 4} \cos \{\mu(x-a)\} e^{-|t|(x-a)}-\left(a_{j 3} / \mu\right) \sin \{\mu(x-a)\} e^{-|t|(x-a)} \\
& -\frac{1}{\mu} \int_{a}^{x}\left\{r(y) g_{j}(y)+q(y) h_{j}(y)\right\} e^{-|t|(x-y)} \sin \{\mu(x-y)\} d y .
\end{aligned}
$$

Let

$$
\begin{aligned}
A & =\max \left(\left|a_{j k}\right|\right) \quad(j=1,2, k=1,2,3,4), \\
H(x) & =\max (|p(x)|,|q(x)|,|r(x)|) ;
\end{aligned}
$$

then

$$
\left|g_{j}(x)\right|,\left|h_{j}(x)\right| \leqq A+\frac{A}{|\mu|}+\frac{1}{|\mu|} \int_{a}^{x}\left\{H(y)\left|g_{j}(y)\right|+H(y)\left|h_{j}(y)\right|\right\} d y .
$$

By Conte and Sangren's lemma [2, p. 700], we obtain

$$
\left|g_{j}(x)\right|,\left|h_{j}(x)\right| \leqq\left\{A+\frac{A}{|\mu|}\right\} \exp \left\{\frac{2}{|\mu|} \int_{a}^{x} H(y) d y\right\} .
$$

It follows that, since $H(x)$ is bounded in $[a, b], g_{j}(x)$ and $h_{j}(x)$ are bounded for $a \leqq x \leqq b$ and $|\mu| \geqq\left|\mu_{0}\right|$. Hence the result (2.3) follows.

By similar arguments the result (2.5) also follows.

Other results follow on putting $u_{j}$ and $v_{j}$ from (2.3) and (2.5) in (2.1) and (2.2).

LEMMA 2.3. If the primes denote differentiation with respect to $x$ and conditions of Lemma 2.2 are satisfied, then

(i) $\phi_{j}^{\prime}(a \mid x, \hat{\lambda})=O\left(|\mu| e^{|t|(x-a)}\right) \quad(j=1,2)$,

(ii) $\quad \begin{aligned} & u_{j}^{\prime}(a \mid x, \lambda)=-a_{j 2} \mu \sin \{\mu(x-a)\}+O\left(e^{|t|(x-a)}\right), \\ & v_{j}^{\prime}(a \mid x, \lambda)=-a_{j 4} \mu \sin \{\mu(x-a)\}+O\left(e^{|t|(x-a)}\right),\end{aligned}(j=1,2)$

(iii) $\phi_{k}^{\prime}(b \mid x, \lambda)=O\left(|\mu| e^{|t|(b-x)}\right) \quad(k=3,4)$,

(iv) $u_{k}^{\prime}(b \mid x, \lambda)=b_{j 2} \mu \sin \{\mu(b-x)\}+O\left(e^{|t|(b-x)}\right)$, $v_{k}^{\prime}(b \mid x, \lambda)=b_{j 4} \mu \sin \{\mu(b-x)\}+O\left(e^{|t|(b-x)}\right)$,

when $k=3, j=1$ and when $k=4, j=2$.

These results are proved by differentiating the expressions for $u_{j}(a \mid x, \lambda)$, etc. obtained from (2.1) and (2.2) and then using Lemma 2.2. 
LEMMA 2.4. If the conditions of Lemma 2.2 are satisfied, then

$$
\left[\phi_{j}(a \mid x, \lambda) \phi_{k}(b \mid x, \lambda)\right]
$$

$$
=-\mu\left(a_{j 2} b_{r 2}+a_{j 4} b_{r 4}\right) \sin \{\mu(b-a)\}+O\left(e^{|t|(b-a)}\right)
$$

where $j=1,2$; when $k=3, r=1$ and when $k=4, r=2$,

$$
\begin{aligned}
W(\lambda)= & \mu^{2}\left(a_{12} a_{24}-a_{14} a_{22}\right)\left(b_{12} b_{24}-b_{14} b_{22}\right) \sin ^{2}\{\mu(b-a)\} \\
& +O\left(|\mu| e^{2|t|(b-a)}\right) .
\end{aligned}
$$

(i) Since $\left[\phi_{1} \phi_{3}\right]$ is independent of $x$, without loss of generality we can evaluate it at $x=b$. Putting the values of $u_{1}(a \mid b, \lambda), u_{1}^{\prime}(a \mid b, \lambda)$, etc. from Lemmas 2.2 and 2.3 in the expression for $\left[\phi_{1} \phi_{3}\right]$, the result for $\left[\phi_{1} \phi_{3}\right]$ follows after some simplification.

By similar arguments the other results follow.

We note that $\left[\phi_{j} \phi_{k}\right]$ are not identically zero. Actually they are integral functions of $\mu$ of order 1 , and so integral functions of $\lambda$ of order $\frac{1}{2}$.

(ii) Substituting for $\left[\phi_{j} \phi_{k}\right]$ from (2.11) in the expression

the result follows.

$$
W(\lambda)=\left[\phi_{1} \phi_{3}\right]\left[\phi_{2} \phi_{4}\right]-\left[\phi_{1} \phi_{4}\right]\left[\phi_{2} \phi_{3}\right]
$$

We note that $W(\lambda)$ is also not identically zero and it possesses an infinity of zeros.

By arguments similar to those in Titchmarsh [4, p. 19, §1.12], it follows that the zeros of $W(\lambda)$ are asymptotic to the zeros of $\mu^{2} \sin ^{2}\{\mu(b-a)\}$, that is to the points where $\mu=n \pi /(b-a)$ for large $|\mu|$ and $|n|$.

For large values of $|n|$ the eigenvalues are asymptotic to $n^{2} \pi^{2} /(b-a)^{2}$.

THEOREM. Let $f(x)=\left(\begin{array}{l}f_{1}(x) \\ f_{2}(x)\end{array}\right)$ be integrable over $(a, b)$. Then the series $\sum_{0}^{\infty} C_{n} \psi_{1 n}(x)$ and $\sum_{0}^{\infty} C_{n} \psi_{2 n}(x)$ for the boundary value problem (1.2) behave as regards convergence in the same way as an ordinary Fourier series. In particular, if $f_{1}(x), f_{2}(x)$ are of bounded variation in the neighbourhood of $x$, they converge to

$$
\frac{1}{2}\left\{f_{1}(x+0)+f_{1}(x-0)\right\} \text { and } \frac{1}{2}\left\{f_{2}(x+0)+f_{2}(x-0)\right\}
$$

respectively.

Consider the integrals

$$
\frac{1}{2 \pi i} \int \Phi_{1}(x, \lambda) d \lambda
$$

and

$$
\frac{1}{2 \pi i} \int \Phi_{2}(x, \lambda) d \lambda
$$


taken round a large closed contour in the $\lambda$-plane, where

is given by

$$
\Phi(x, \lambda)=\left(\begin{array}{l}
\Phi_{1}(x, \lambda) \\
\Phi_{2}(x, \lambda)
\end{array}\right)
$$

$$
\Phi(x, \hat{\lambda})=-\int_{a}^{b}\left(G_{i j}(x, y ; \hat{\lambda})\right) f(y) d y .
$$

By the residue theorem, (2.13) and (2.14) are respectively $\sum_{n=0}^{m} C_{n} \psi_{1 n}(x)$ and $\sum_{n=0}^{m} C_{n} \psi_{2 n}(x)$, the summation being taken over those values of $m$ for which the poles of $\Phi(x, \lambda)$ lie in the contour taken.

As in Titchmarsh [4, Theorem 1.9, p. 12], let $\lambda=\mu^{2}, \mu=\sigma+i$ and let the upper half of the $\lambda$-contour correspond to the quarter square in the $\mu$-plane made up by the lines

$$
\begin{aligned}
\sigma=\left(n+\frac{1}{2}\right) \pi /(b-a) & \left(0 \leqq t \leqq\left(n+\frac{1}{2}\right) \pi /(b-a)\right), \\
t=\left(n+\frac{1}{2}\right) \pi /(b-a) & \left(0 \leqq \sigma \leqq\left(n+\frac{1}{2}\right) \pi /(b-a)\right),
\end{aligned}
$$

and let the $\lambda$-contour be symmetrical about the real axis.

Since $|\sin \{\mu(b-a)\}|>A e^{t(b-a)}$, it follows from (2.12) that

$$
\begin{aligned}
\frac{1}{W(\lambda)}= & \frac{1}{\mu^{2}\left(a_{12} a_{24}-a_{14} a_{22}\right)\left(b_{12} b_{24}-b_{14} b_{22}\right) \sin ^{2}\{\mu(b-a)\}} \\
& \times\left\{1+O\left(\frac{1}{|\mu|}\right)\right\} .
\end{aligned}
$$

We have, for $y \in[a, x)$,

$$
\begin{aligned}
G_{11}(x, y ; \lambda)= & \psi_{11}(x, \lambda) u_{1}(y, \lambda)+\psi_{21}(x, \lambda) u_{2}(y, \lambda) \\
= & {[W(\lambda)]^{-1}\left\{\left(\left[\phi_{2} \phi_{4}\right] u_{3}(b \mid x, \lambda)-\left[\phi_{2} \phi_{3}\right] u_{4}(b \mid x, \lambda)\right) u_{1}(y, \lambda)\right.} \\
& \left.+\left(\left[\phi_{1} \phi_{3}\right] u_{4}(b \mid x, \lambda)-\left[\phi_{1} \phi_{4}\right] u_{3}(b \mid x, \lambda)\right) u_{2}(y, \lambda)\right\} .
\end{aligned}
$$

If $\Delta$ denotes the expression in brackets \{\} , we get, by using (2.4), (2.6) and (2.11),

$$
\begin{aligned}
\Delta= & \mu\left(a_{12} a_{24}-a_{14} a_{22}\right)\left(b_{14} b_{22}-b_{12} b_{24}\right) \\
& \times \sin \{\mu(b-a)\} \cos \{\mu(b-x)\} \cos \{\mu(y-a)\} \\
& +O(\exp (t[2(b-a)+y-x]))
\end{aligned}
$$

Therefore

$$
G_{11}(x, y ; \lambda)=-\frac{\cos \{\mu(b-x)\} \cos \{\mu(y-a)\}}{\mu \sin \{\mu(b-a)\}}+O\left(\frac{e^{t(y-x)}}{|\mu|^{2}}\right)
$$


Similarly

$$
\begin{aligned}
& \text { (2.16) } \quad G_{12}(x, y ; \lambda)=O\left(\frac{e^{t(y-x)}}{|\mu|^{2}}\right) . \\
& \text { (2.17) } G_{21}(x, y ; \lambda)=O\left(\frac{e^{t(y-x)}}{|\mu|^{2}}\right) . \\
& \text { (2.18) } \quad G_{22}(x, y ; \lambda)=-\frac{\cos \{\mu(b-x)\} \cos \{\mu(y-a)\}}{\mu \sin \{\mu(b-a)\}}+O\left(\frac{e^{t(y-x)}}{|\mu|^{2}}\right) .
\end{aligned}
$$

Hence contribution towards $\Phi_{1}(x, \lambda)$ for the limit $a$ to $x$ is given by

$$
\begin{aligned}
\int_{a}^{x} \frac{\cos \{\mu(b-x)\} \cos \{\mu(y-a)\}}{\mu \sin \{\mu(b-a)\}} & f_{1}(y) d y \\
+ & o\left\{\frac{1}{|\mu|^{2}} \int_{a}^{x} e^{t(y-x)}\left(\left|f_{1}(y)\right|+\left|f_{2}(y)\right|\right) d y\right\} .
\end{aligned}
$$

Similarly for the limits from $x$ to $b$. A similar result holds for $\Phi_{2}(x, \lambda)$.

The theorem now follows by proceeding in an exactly similar way as that in Titchmarsh [4, Theorem 1.9, p. 12].

\section{REFERENCES}

1. B. Bhagat, Eigenfunction expansions associated with a pair of secondorder differential equations, Proc. Nat. Inst. Sci. India Part A 35 (1969), 161-174. MR 40 \#2949.

2. S. D. Conte and W. C. Sangren, An asymptotic solution for a pair of first order equations, Proc. Amer. Math. Soc. 4 (1953), 696-702. MR 15, 314.

3. B. W. Roos and W. C. Sangren, Asymptotic solutions and an equiconvergence theorem for a pair of first-order differential equations, J. Soc. Indust. Appl. Math. 11 (1963), 421-430. MR 28 \#1344.

4. E. C. Titchmarsh, Eigenfunction expansions associated with second-order differential equations. Part I, 2nd ed., Clarendon Press, Oxford, 1962. MR 31 \#426.

Science College, Patna University, Patna 5, India 